\title{
Phase I study of preoperative chemoradiotherapy with sequential oxaliplatin and irinotecan with S-1 for locally advanced rectal cancer
}

\author{
HIROYUKI FUJIKAWA, YUJI TOIYAMA, YASUHIRO INOUE, YUSUKE OMURA, SHOZO IDE, \\ TAKAHITO KITAJIMA, HIROMI YASUDA, YOSHINAGA OKUGAWA, YOSHIKI OKITA, \\ SHIGEYUKI YOSHIYAMA, JUNICHIRO HIRO, MINAKO KOBAYASHI, MASAKI OHI, \\ TOSHIMITSU ARAKI and MASATO KUSUNOKI
}

\begin{abstract}
Department of Gastrointestinal and Pediatric Surgery, Division of Reparative Medicine, Institute of Life Sciences, Mie University Graduate School of Medicine, Tsu, Mie 514-8507, Japan
\end{abstract}

Received October 16, 2018; Accepted January 17, 2019

DOI: 10.3892/ol.2019.10028

\begin{abstract}
The present study designed a novel preoperative chemoradiotherapy (CRT) with sequential oxaliplatin and irinotecan with S-1 for locally advanced rectal cancer (LARC). This phase I study evaluated the maximum tolerated dose and recommended dose (RD) of oxaliplatin following irinotecan with S-1. Patients with clinical stage T3 or 4 or involvement of the regional nodes and no evidence of distant metastases were treated with fixed doses of $\mathrm{S}-1\left(80 \mathrm{mg} / \mathrm{m}^{2} /\right.$ day $)$ on days $1-5$, $8-12,15-19,22-27$ and $29-33$, and irinotecan $\left(40 \mathrm{mg} / \mathrm{m}^{2} /\right.$ day) on days 1 and 8 , followed by oxaliplatin on days 22 and 29 . The dose of oxaliplatin was initially $40 \mathrm{mg} / \mathrm{m}^{2}$ (level 1) with a predefined dose escalation schedule. The radiation dose was $1.8 \mathrm{~Gy} /$ fraction to a total dose of $45 \mathrm{~Gy}$. A total of 9 patients were enrolled in the present study and 7 patients completely received CRT with this study protocol. The maximum tolerated dose for oxaliplatin was $50 \mathrm{mg} / \mathrm{m}^{2}$ (level 2). Three of four patients experienced dose-limiting toxicity (grade 3 diarrhea) in oxaliplatin phase of level 2 dose. The RD of oxaliplatin was $40 \mathrm{mg} / \mathrm{m}^{2}$ (level 1 dose). In addition, 2 patients had pathological CR (28.5\%). Novel preoperative CRT with sequential oxaliplatin and irinotecan with S-1 for LARC resulted in acceptable toxicity and promising efficacy. However, the RD of oxaliplatin was lower than in previous CRT studies that combined oxaliplatin with S-1. To administer higher oxaliplatin, we have planned a phase I trial of preoperative CRT with sequential oxaliplatin followed by irinotecan with S-1 for LARC.
\end{abstract}

Correspondence to: Dr Hiroyuki Fujikawa, Department of Gastrointestinal and Pediatric Surgery, Division of Reparative Medicine, Institute of Life Sciences, Mie University Graduate School of Medicine, 2-174 Edobashi, Tsu, Mie 514-8507, Japan E-mail: f0609@clin.medic.mie-u.ac.jp

Key words: rectal cancer, chemoradiotherapy, S-1, oxaliplatin, irinotecan

\section{Introduction}

Preoperative chemoradiotherapy (CRT) significantly reduces the risk of local recurrence and cancer-specific mortality compared with surgery alone in locally advanced rectal cancer (LARC) $(1,2)$. Following a German phase III trial in 2004, preoperative CRT with infusional 5-florouracil (5-FU) and total mesorectal excision surgery has become the standard treatment for stage II and III rectal cancer in Western countries (2). Recently, new agents such as oral fluoropyrimidines, oxaliplatin and irinotecan, which were used in the metastatic disease setting or adjuvant chemotherapy, have been used by several groups to modify tumor response in clinical trials of CRT (3). CAO/ARO/AIO-04 phase III trials showed that adding oxaliplatin to 5-FU improved pathological complete response (pCR) and disease free survival (DFS) compared with 5-FU alone (4), whereas STAR-01, ACCORD 12 and NSABP R-04 phase III trials with 5-FU or capecitabine plus oxaliplatin did not show significant improvements in pCR and DFS (5-7). In addition, phase III trials with irinotecan have not been reported, but early phase I/II trials with 5-FU or capecitabine plus irinotecan showed that $\mathrm{pCR}$ rates were $13.7-37 \%$ (8-13). Therefore, the use of fluoropyrimidine plus oxaliplatin or irinotecan in CRT is not recommended outside of clinical trials.

S-1 is an oral fluoropyrimidine containing tegafur, gimeracil, and oteracil potassium in a molar ratio of 1:0.4:1 (14). Tegafur is a prodrug of 5-FU, and gimeracil is a reversible inhibitor of dihydropyrimidine dehydrogenase that degrades 5-FU (15). Oteracil potassium inhibits the enzyme orotate phosphoribosyl-transferase, which converts tegafur to 5-FU and decreases gastrointestinal toxicity of 5-FU (15). S-1 has good anticancer efficacy for colorectal cancer (CRC) and an acceptable toxicity profile (16). In addition, chemoradiotherapy with $\mathrm{S}-1$ was effective and well tolerated in a previous phase I/II study (17). Early phase studies of preoperative CRT with S-1 plus irinotecan (phase II) or oxaliplatin (phase II) regimen showed favorable toxicity profile and good pCR rates $(18,19)$. 
Recently, triplet combination chemotherapy regimen (FOLFOXRI) has been demonstrated to be superior to doublet regimen (FOLFIRI) in metastatic CRC, though triplet regimen has more adverse effects than doublet chemotherapy (20). Several tumors, including CRC, have intra-tumor genetic heterogeneity, which reflects the presence of different subclonal populations within the cancer and are likely associated with clinical course and response to therapy $(21,22)$. Chemotherapy or chemoradiotherapy, including more agents with different mechanisms, may improve treatment response in view of this heterogeneity. Therefore, we hypothesized that chemoradiation with triplet radiosensitizer of fluoropyrimidines, oxaliplatin and irinotecan may have a higher response than regimens used in previous studies. However, the feasibility of chemoradiation with triplet radiosensitizer of fluoropyrimidines, oxaliplatin and irinotecan is not well known. Therefore, we designed a new preoperative CRT with sequential oxaliplatin and irinotecan with S-1 for LARC and aimed to determine the maximum tolerated dose (MTD) and recommended dose (RD) of oxaliplatin following irinotecan in a phase I study.

\section{Materials and methods}

Ethics and patient consent. The present study was reviewed and approved by Mie University Institutional Review Board, and the study was performed in accordance with the Helsinki Declaration of 1975, as revised in 2000. Patients were required to provide written informed consent prior to enrollment. The present study was registered at the UMIN Clinical Trial Registry as UMIN000017674 (further details accessible at: http://www.umin.ac.jp/ctr/index.htm).

Eligibility criteria. Eligible patients had LARC with T3 to 4 or involvement of regional nodes as determined by computed tomography (CT), magnetic resonance imaging (MRI), or endoscopic ultrasound and had histologically-confirmed adenocarcinoma prior to surgery. Eligible patients also had an Eastern Cooperative Oncology Group performance status of $0-1$ and a survival expectation of $>3$ months. Additional eligibility criteria included: age 20-80 years at enrollment, no severe compromise of main organ functions (including bone marrow, lung, liver and kidney), and blood specific biochemistry results (leukocyte count 4,000-12,000/ $\mu$, platelet count $\geq 100 \times 10^{3} / \mu 1$, hemoglobin concentration $\geq 9.0 \mathrm{~g} / \mathrm{dl}$, total bilirubin concentration $\leq 1.5 \mathrm{x}$ upper normal limit, serum aspartate aminotransferase and alanine aminotransferase levels $<2.5 x$ upper normal limit, and serum creatinine concentration $<1.5 x$ upper normal limit). The exclusion criteria were patients with potential risk factors for $\mathrm{S}-1$, irinotecan, and oxaliplatin-related adverse events. The risk factors included patients with child-bearing potential or lactation; patients without intention to use contraception; clinically significant cardiovascular, pulmonary or renal disease; clinical evidence of gastrointestinal bleeding; bowel obstruction and perforation; active inflammatory bowel disease; active infection; considerable pleural effusion, cardiac effusion and ascites; and any other cases regarded as inadequate for enrollment by the investigator.

Pretreatment evaluation. Complete clinical and radiographic staging was performed prior to enrollment in the study.
Patients underwent the following evaluations: History and physical examination; CT scan of the chest, abdomen, and pelvis; chest X-ray; colonoscopy; barium enema; endoscopic ultrasound; and pelvic MRI. A complete blood count with differential analysis, serum chemistry tests, urinalysis, electrocardiogram, carcinoembryonic antigen, and carbohydrate antigen 19-9 levels were obtained. During treatment, patients were evaluated at least weekly via a history and physical examination, and they underwent a complete blood count with differential analysis, electrolyte analysis, liver function tests, chemistry panel assays, coagulation panel assays, and urinalysis.

Treatment. Patients underwent four-field (anterior-posterior, posterior-anterior, and right and left laterals) approach and radiation therapy was delivered using a 10-MV linear accelerator. Patients were treated in the prone position using a dedicated device to minimize exposure of the small bowel. A CT-based treatment planning system was mandatory to define the planning target volume, including the primary tumor, internal iliac lymph nodes, presacral lymph nodes, obturator lymph nodes, and the surrounding mesorectum. Radiotherapy was administered in fractions of $1.8 \mathrm{~Gy} /$ day, 5 days/week, for 5 weeks. The total dose of radiation delivered was 45 Gy. During radiation therapy, patients received chemotherapy using the following regimen. The regimen comprised fix doses of $\mathrm{S}-1\left(80 \mathrm{mg} / \mathrm{m}^{2} /\right.$ day $)$ and irinotecan (40 $\mathrm{mg} / \mathrm{m}^{2} /$ day). S-1 was given on days $1-5,8-12,15-19$, 22-26 and $29-33$. Irinotecan $\left(40 \mathrm{mg} / \mathrm{m}^{2} /\right.$ day) was given on days 1 and 8, and oxaliplatin was given on days 22 and 29, 2 weeks after irinotecan administration. Table I provides a summary of oxaliplatin dose levels. Oxaliplatin administration was initiated at $40 \mathrm{mg} / \mathrm{m}^{2}$ (level 1), with a planned dose escalation to dose levels 2 and 3. A treatment schema is shown in Fig. 1. Patients were premedicated prior to chemotherapy treatment to minimize nausea and vomiting, which are associated with 5-HT3 receptor antagonists. The interval between the completion of CRT and operation was 6-8 weeks. The assessment of tumor resectability included a history and physical examination, imaging evaluation using CT and MRI, and proctosigmoidoscopy. Following thorough exploration of the peritoneal cavity and distant lesions for metastases, low anterior resection or abdominoperineal resection with total mesorectal excision was performed in conjunction with a colonic J-pouch-anal anastomosis (CAA). A diverting ileostomy was usually used to prevent from anastomotic leakage.

Assessment. Adverse events were assessed at least weekly during radiation therapy, using the National Cancer Institute common toxicity criteria (version 3.0). Side effects were managed aggressively with standard supportive measures. Resectability was also evaluated and a complete pathological response was defined as no evidence of malignancy in the specimen.

Study design, definitions, and endpoints. The primary endpoint of this study was the determination of the MTD and the RD. The secondary endpoint was evaluation of the extent and frequency of the adverse events and the resectability of locally-advanced 
rectal cancer. The dose-limiting toxicity (DLT) was defined as Grade 4 hematological toxicity (leucopenia and neutropenia persisting beyond 4 days and thrombocytopenia); Grade 3 or higher non-hematological toxicity, despite adequate supportive care; any single interruption of radiation therapy of $\geq 7$ days or $>2$ interruptions per radiation course; any delay of $>14$ days in the completion of radiation therapy; and any treatment-related hospitalization or death. Perianal dermatitis was defined as adverse effects of radiation and excluded as a criterion of DLT. Patients with DLT before oxaliplatin administration were not included in this study. The MTD was defied as the dose level that produced DLT in at least two out of three patients, or two out of six patients. If DLT occurred in one of the first three patients, three additional patients were assigned to receive the same dose level. If none of the three patients initially receiving a given dose level exhibited DLT, or if one out of six patients exhibited DLT, the dose was increased to the next level. Dose escalation was not allowed in the same patient. The dose level immediately below the MTD was considered the RD for phase II studies.

\section{Results}

Patient characteristics. From May 2015 through February 2017, nine patients treated at our institution were enrolled in this study. Two patients who developed Grade 3 radiation enterocolitis before oxaliplatin administration were not included in this study because of delays of $>14$ days in completing radiation therapy. Characteristics of patients who completely received this protocol treatment are summarized in Table II.

DLT and RD levels. The observed toxicities are described in Table III. Three patients were initially enrolled at dose level 1. In the $\mathrm{S}-1+$ irinotecan regimen on level 1 protocol phase, almost adverse effects were Grade 1 though two patients had nausea of Grade 2 which required intravenous fluids. All three patients had Grade 1 perianal dermatitis by first dose of S-1+oxaliplatin. In the level $1 \mathrm{~S}-1+$ oxaliplatin regimen phase, one patient experienced Grade 2 diarrhea and one patient developed Grade 2 anemia from Grade 1 in the irinotecan phase. In addition, all three patients developed perianal dermatitis by completion of irradiation. In summary of the level 1 protocol, all three patients were treated without interruptions to chemotherapy or irradiation or Grade 3-4 adverse effects defined as DLT. Second, three patients were enrolled at dose level 2. In phase of S-1+irinotecan regimen on level 2 protocol, one patient experienced a 3-day interruption of irradiation because of developing Grade 3 diarrhea after the second dose of irinotecan. Another patient also had a 4-day interruption of irradiation with Grade 2 stomatitis, fatigue, and diarrhea just before the first dose of S-1+oxaliplatin. All three patients had Grade 1 perianal dermatitis caused by the first dose of S-1+oxaliplatin as well as the $\mathrm{S}-1+$ irinotecan regimen on level 1 protocol phase. In the level $2 \mathrm{~S}-1+$ oxaliplatin regimen phase, two patients developed Grade 3 diarrhea treated with intravenous fluids just before completion of irradiation though irradiation was not interrupted. Grade 1 peripheral neuropathy caused by oxaliplatin was observed in all three patients. Subsequently, three additional patients were enrolled at the same dose level 1 according to the study protocol, because of
Table I. Summary of S-1/CPT-11/L-OHP dose levels used in combination with radiation therapy.

\begin{tabular}{lccc}
\hline Level & S-1 $(\mathrm{mg} / \mathrm{m})$ & CPT-11 $\left(\mathrm{mg} / \mathrm{m}^{2}\right)$ & L-OHP $\left(\mathrm{mg} / \mathrm{m}^{2}\right)$ \\
\hline-1 & 80 & 40 & 30 \\
1 & 80 & 40 & 40 \\
2 & 80 & 40 & 50 \\
3 & 80 & 40 & 60 \\
\hline
\end{tabular}

CPT-11, irinotecan; L-OHP, oxaliplatin.

Table II. Patient characteristics.

\begin{tabular}{lc}
\hline Characteristics & Patients \\
\hline Age (range) & $55(38-76)$ \\
Sex & 3 \\
Male & 4 \\
Female & \\
Performance status & 7 \\
0 & \\
Tumor site & 5 \\
Rb & 2 \\
Rb-P & \\
Clinical stage & \\
cT2N1 & 1 \\
cT3N0 & 1 \\
cT3N1 & 1 \\
cT3N2 & \\
cT4N1 & 1 \\
cT4N2 & 1 \\
\hline
\end{tabular}

$\mathrm{Rb}$, rectum below the peritoneal reflection; $\mathrm{P}$, anal canal.

incidence of Grade 3 non-hematological toxicity. One patient had Grade 2 diarrhea without interruption of irradiation and chemotherapy in the $\mathrm{S}-1+$ irinotecan regimen phase. In addition, this patient experienced Grade 3 diarrhea and stomatitis with cessation of radiation therapy (total $43.2 \mathrm{~Gy}$ ), delay of chemotherapy and administration of intravenous fluids in the $\mathrm{S}-1+$ oxaliplatin regimen phase. Because non-hematological toxicity as DLT was observed in three patients and level 2 was designated as MTD, this study finished by enrolling seven patients. Therefore, level 1 was considered the RD for the phase II study.

Efficacymeasures. We evaluated the tumor response to chemoradiotherapy. Details of the patients' treatment responses are shown in Table IV. The downstaging rate in the tumor and nodal stage was $85.7 \%$ (6/7 patients) and 57.1\% (4/7 patients), respectively. Five patients achieved radiographic CR (28.5\%) and PR (42.8\%). Five patients underwent laparoscopic low anterior resection with colonic J-pouch reconstruction, one underwent laparoscopic CAA with colonic J-pouch and total intersphincteric resection, and one underwent laparoscopic 
Table III. Toxicity parameters evaluated in the study and matched control group.

\begin{tabular}{|c|c|c|c|c|c|c|c|c|}
\hline \multirow[b]{2}{*}{ S-1+CPT11 toxicity } & \multicolumn{4}{|c|}{ Dose level 1} & \multicolumn{4}{|c|}{ Dose level 2} \\
\hline & G1 & $\mathrm{G} 2$ & G3 & G4 & G1 & $\mathrm{G} 2$ & G3 & G4 \\
\hline \multicolumn{9}{|l|}{ Hematological } \\
\hline Neutropenia & 0 & 0 & 0 & 0 & 2 & 0 & 0 & 0 \\
\hline Anemia & 1 & 0 & 0 & 0 & 0 & 0 & 0 & 0 \\
\hline Thrombocytopenia & 0 & 0 & 0 & 0 & 0 & 0 & 0 & 0 \\
\hline AST/ALT abnormalities & 1 & 0 & 0 & 0 & 1 & 0 & 0 & 0 \\
\hline Hyperbilirubinemia & 0 & 0 & 0 & 0 & 0 & 0 & 0 & 0 \\
\hline \multicolumn{9}{|l|}{ Non-hematological } \\
\hline Nausea & 0 & 2 & 0 & 0 & 0 & 0 & 0 & 0 \\
\hline Vomiting & 0 & 0 & 0 & 0 & 0 & 0 & 0 & 0 \\
\hline Stomatitis & 1 & 0 & 0 & 0 & 1 & 1 & 0 & 0 \\
\hline Fatigue & 1 & 0 & 0 & 0 & 2 & 1 & 0 & 0 \\
\hline Diarrhea & 1 & 0 & 0 & 0 & 1 & 1 & 1 & 0 \\
\hline Peripheral neuropathy & 0 & 0 & 0 & 0 & 0 & 0 & 0 & 0 \\
\hline \multirow[t]{2}{*}{ Skin (perianal dermatitis) } & 3 & 0 & 0 & 0 & 3 & 0 & 0 & 0 \\
\hline & \multicolumn{4}{|c|}{ Dose level 1} & \multicolumn{4}{|c|}{ Dose level 2} \\
\hline S-1+L-OHP toxicity & G1 & $\mathrm{G} 2$ & G3 & G4 & G1 & $\mathrm{G} 2$ & G3 & G4 \\
\hline \multicolumn{9}{|l|}{ Hematological } \\
\hline Neutropenia & 1 & 0 & 0 & 0 & 0 & 1 & 0 & 0 \\
\hline Anemia & 0 & 1 & 0 & 0 & 0 & 0 & 0 & 0 \\
\hline Thrombocytopenia & 0 & 0 & 0 & 0 & 0 & 0 & 0 & 0 \\
\hline AST/ALT abnormalities & 2 & 0 & 0 & 0 & 3 & 0 & 0 & 0 \\
\hline Hyperbilirubinemia & 0 & 0 & 0 & 0 & 0 & 0 & 0 & 0 \\
\hline \multicolumn{9}{|l|}{ Non-hematological } \\
\hline Nausea & 0 & 0 & 0 & 0 & 0 & 1 & 0 & 0 \\
\hline Vomiting & 0 & 0 & 0 & 0 & 0 & 0 & 0 & 0 \\
\hline Stomatitis & 0 & 0 & 0 & 0 & 0 & 1 & 0 & 0 \\
\hline Fatigue & 1 & 0 & 0 & 0 & 1 & 1 & 0 & 0 \\
\hline Diarrhea & 2 & 1 & 0 & 0 & 0 & 1 & 3 & 0 \\
\hline Peripheral neuropathy & 0 & 0 & 0 & 0 & 2 & 0 & 0 & 0 \\
\hline Skin (perianal dermatitis) & 0 & 3 & 0 & 0 & 1 & 3 & 0 & 0 \\
\hline
\end{tabular}

CPT-11, irinotecan; L-OHP, oxaliplatin.

Concomitant chemoradiotherapy with sequential regimen of S-1, CPT11 and L-OHP in patients with locally advanced rectal cancer

\begin{tabular}{lllllll} 
Day & 1 & 8 & 15 & 22 & 29 & 33 \\
\hline $\mathrm{RT}(1.8 \mathrm{~Gy} / \mathrm{day})$ & $\uparrow \uparrow \uparrow \uparrow \uparrow$ & $\uparrow \uparrow \uparrow \uparrow \uparrow$ & $\uparrow \uparrow \uparrow \uparrow \uparrow$ & $\uparrow \uparrow \uparrow \uparrow \uparrow$ & $\uparrow \uparrow \uparrow \uparrow \uparrow$ \\
$\mathrm{S}-1\left(80 \mathrm{mg} / \mathrm{m}^{2}\right)$ & $\uparrow \uparrow \uparrow \uparrow \uparrow$ & $\uparrow \uparrow \uparrow \uparrow \uparrow$ & $\uparrow \uparrow \uparrow \uparrow \uparrow$ & $\uparrow \uparrow \uparrow \uparrow \uparrow$ & $\uparrow \uparrow \uparrow \uparrow \uparrow$ \\
CPT-11 $\left(40 \mathrm{mg} / \mathrm{m}^{2}\right)$ & $\uparrow$ & $\uparrow$ & & $\uparrow$ & $\uparrow$ \\
L-OHP & & & & $\uparrow$ & $\uparrow$
\end{tabular}

Figure 1. Concomitant chemoradiotherapy with sequential regimen of S-1, CPT-11 and L-OHP in patients with locally advanced rectal cancer. CPT-11 with S-1 was sequentially followed by L-OHP with S-1. CPT-11, irinotecan; L-OHP, oxaliplatin.

CAA with coloplasty and subtotal intersphincteric resection. Surgical site infection and anastomotic leakage were not observed. Two patients had a microscopically-positive circumferential resectional margin (R1) and five had complete resection (R0). Finally, two patients had pathological CR $(28.5 \%)$. 
Table IV. Individual patient characteristics and treatment response.

\begin{tabular}{lccccccc}
\hline Patient & $\begin{array}{c}\text { Dose } \\
\text { level }\end{array}$ & $\begin{array}{c}\text { Pretreatment } \\
\text { stage }\end{array}$ & $\begin{array}{c}\text { Pathological } \\
\text { stage }\end{array}$ & $\begin{array}{c}\text { Dose-limiting } \\
\text { toxicity }\end{array}$ & $\begin{array}{c}\text { Radiographic } \\
\text { response }\end{array}$ & $\begin{array}{c}\text { Pathological } \\
\text { effects grade }^{\text {a }}\end{array}$ & $\begin{array}{c}\text { Surgical } \\
\text { outcome }^{-}\end{array}$ \\
\hline 1 & 1 & T3N1M0 & T0NOM0 & No & CR & $3^{\mathrm{b}}$ & R0 \\
2 & 1 & T4N2M0 & T2N0M0 & No & PR & 2 & R0 \\
3 & 1 & T4N2M0 & T2N1M0 & No & SD & $1 \mathrm{~b}$ & R0 \\
4 & 2 & T2N1M0 & T0N0M0 & Yes & CR & $3^{\text {b }}$ & R0 \\
5 & 2 & T3N0M0 & T3N0M0 & No & SD & $1 \mathrm{a}$ & R1 \\
6 & 2 & T4N2M0 & T3N2M0 & Yes & PR & $1 \mathrm{a}$ & R1 \\
7 & 2 & T3N2M0 & T2N2M0 & Yes & PR & $1 \mathrm{a}$ & R0 \\
\hline
\end{tabular}

${ }^{a}$ The degree of histopathological tumor regression based on the Guidelines for Clinical and Pathological Studies on Carcinoma of the Colon and Rectum: Grade 1a, >2/3 vital residual tumor cells (VRTCs); Grade 1b, 1/32/3 VRTCs; Grade 2, <1/3 VRTSCs; and Grade 3, no VRTCs. ${ }^{b} \mathrm{pCR}$, pathologic complete response; PR, partial response; SD, stable disease; R1, microscopically positive resection margin; R0, microscopically negative resection margin.

\section{Discussion}

CRT is an important element that enhances local control for patients with LARC such as T3, T4, and lymph node metastasis (3). Proposed improvement for CRT have been better local control and less toxicity. Preoperative CRT improved local control and reduced therapeutic toxicity compared with postoperative CRT and preoperative therapy with continuous infusional 5-FU is the standard practice for LARC (2). Furthermore, many clinical trials have been performed to improve clinical outcomes in preoperative CRT (3). Newer generation agents, including cytotoxic and biological drugs such as oral fluoropyrimidines, oxaliplatin, irinotecan, antibody against vascular endothelial growth factor, and anti-epidermal growth factor receptor, have been used as radiosensitizers in recent clinical trials (3). Despite the oncological benefits of these agents shown in clinical trials, the mechanism of conventional chemotherapeutic agents for radiosensitization varies among the different agents (23). One mechanism of 5-FU as radiosensitizer is through killing S phase cells, which are relatively radioresistant (24). Oxaliplatin plays a role as a radiosensitizer through various mechanisms, such as DNA damage by the formation of inter- and intra-strand crosslinks, induction of G2 and M cell cycle arrest, and blockage of DNA repair $(25,26)$. Irinotecan is metabolized to SN38, which inhibits topoisomerase I and has radiosensitizing properties. It has been suggested that irinotecan may enhance the lethal effects of ionizing radiation by attaching to the DNA-topoisomerase I adducts in sites of single strand DNA breaks (26). These cytotoxic drugs provide therapeutic efficacy by different mechanisms for each other and combination therapies with these agents are standard chemotherapy in CRC. However, intra-tumor heterogeneity, which reflects the presence of different subclonal populations within the cancer, likely impacts the response and resistance to therapy $(21,22)$. Combination therapy with more agents may be effective countermeasure to drug resistance due to tumor heterogeneity. In addition, the toxicity of triplet regimen is greater while anticancer effect of triplet is superior to doublet in CRC (27). Therefore, we designed chemoradiation with triplet radiosensitizer of fluoropyrimidines, oxaliplatin, and irinotecan and adopted a sequential schedule of oxaliplatin and irinotecan for low toxicity. This sequential administration could reduce adverse effects than simultaneous administration and may be feasible not only for young patients but also for elderly patients.

In this study, we performed CRT with S-1 plus oxaliplatin following irinotecan for seven patients with LARC. The prescribed dose $\left(40,80 \mathrm{mg} / \mathrm{m}^{2}\right)$ of irinotecan and S-1 in this study was based on previous phase II studies (23). Initial dose of irinotecan was prescribed as the minimum dose following these studies $(28,29)$ because this trial was the first study with triple agents: S-1 and irinotecan followed by S-1 and oxaliplatin. A multicenter phase I trial (SAMRAI-1) concluded that the RD of irinotecan was $60 \mathrm{mg} / \mathrm{m}^{2}$ in preoperative CRT combined with S-1 and irinotecan and incidence of Grade 3 or higher diarrhea defined as DLT was $11.1 \%$ (30). Our study showed Grade 3 enterocolitis of two patients, who were accordingly excluded from this trial, and Grade 3 diarrhea of one patient in S-1 plus irinotecan phase despite lower dose and shorter dose duration compared with SAMRAI-1. This discrepancy may be due to UGT1A1 genetic polymorphisms, which delay the metabolism of $\mathrm{SN}-38$, the active metabolite of irinotecan, and are susceptible to toxicities such as neutropenia and diarrhea. Our study did not evaluate UGT1A1 genetic polymorphisms because the dose of irinotecan was far lower than that in doublet regimen of systemic chemotherapy using irinotecan, whereas SAMRAI-1 excluded a genotype of UGT1A $1 * 6 / * 6$, UGT $1 \mathrm{~A} 1 * 28 / * 28$ or heterozygote for both UGT1A $1 * 6$ and $* 28$. In addition, Grade 3 enterocolitis in two patients might be associated with adverse effects of irradiation and chemotherapy.

The RD of oxaliplatin was determined to be $40 \mathrm{mg} / \mathrm{m}^{2}$. DLT occurred in three of seven patients at dose level 2 and all DLTs were Grade 3 diarrhea. However, the RD of oxaliplatin identified in this study was lower than that in previous trials of oral fluoropyrimidines plus oxaliplatin. Phase III trials with capecitabine plus oxaliplatin were performed with oxaliplatin $50-60 \mathrm{mg} / \mathrm{m}^{2}(23)$. In addition, oxaliplatin was also administered with $60 \mathrm{mg} / \mathrm{m}^{2}$ in phase II studies with S-1 plus oxaliplatin (19). The lower RD of oxaliplatin in this study might be caused by administration followed by irinotecan considering that all DLTs were Grade 3 diarrhea. Our protocol, 
combined with S-1 $80 \mathrm{mg} / \mathrm{m}^{2}$, irinotecan $40 \mathrm{mg} / \mathrm{m}^{2}$ and followed by oxaliplatin $40 \mathrm{mg} / \mathrm{m}^{2}$, was safe in this patient group without genotyping of UGT1A1 polymorphisms. However, if sequential CRT regimen with oxaliplatin following irinotecan in this study is transposed to inverse sequential regimen with irinotecan following oxaliplatin, diarrhea may decrease in the late phase of CRT.

With 5-FU or capecitabine plus oxaliplatin CRT, pCR rates were 16-17 and 13.3-19.2\%, respectively, in phase III trials, while the pCR rates of 5-FU or capecitabine plus irinotecan CRT were 13.7-37 and 15-50\%, respectively, in phase II studies (23). In S-1 based CRT regimens of early phase studies, pCR rates of CRT with S-1 plus oxaliplatin and irinotecan were 27.3 and $34.7 \%$, respectively $(18,19)$. Our study showed an acceptable pCR rate of $28.5 \%$ though it was a phase 1 trial. Though we anticipated that a CRT regimen with triplet agents including oxaliplatin, irinotecan, and S-1 could potentially have better pCR rates than previous CRT studies with S-1 plus oxaliplatin or irinotecan, our CRT regimen did not show excellent $\mathrm{pCR}$ rates in this study. This result might be affected according to lower dose of both oxaliplatin and irinotecan than in the SAMRAI-1 study (30) and SHOGUN trial with $\mathrm{S}-1$ (31).

In conclusion, a new preoperative CRT with sequential oxaliplatin and irinotecan with S-1 for LARC resulted in acceptable toxicity and promising efficacy. However, the RDs of oxaliplatin were lower than in previous CRT studies that combined oxaliplatin with S-1. To administer higher oxaliplatin dose, sequential CRT regimen with oxaliplatin followed by irinotecan may be recommended. Therefore, we are planning a phase I trial of preoperative CRT with sequential oxaliplatin followed by irinotecan with S-1 for LARC.

\section{Acknowledgements}

Not applicable.

\section{Funding}

No funding was received.

\section{Availability of data and materials}

All data generated or analyzed during this study are included in this published article.

\section{Authors' contributions}

HF drafted the manuscript. HF, YOm, SI, TK, HY, YOku, YOki, SY, JH, MKo, MO and TA contributed to the collection and analysis of the data. HF, YT, YI and MKu conceived and designed the study, and edited the manuscript. All authors read and approved the final manuscript.

\section{Ethics approval and consent to participate}

The present study was reviewed and approved by Mie University Institutional Review Board (Mie, Japan; approval no. 2892). Patients were required to provide written informed consent prior to enrollment.

\section{Patient consent for publication}

Not applicable.

\section{Competing interests}

The authors declare that they have no competing interests.

\section{References}

1. Sauer R, Liersch T, Merkel S, Fietkau R, Hohenberger W, Hess C Becker H, Raab HR, Villanueva MT, Witzigmann H, et al: Preoperative versus postoperative chemoradiotherapy for locally advanced rectal cancer: Results of the German CAO/ARO/AIO-94 randomized phase III trial after a median follow-up of 11 years. J Clin Oncol 30: 1926-1933, 2012.

2. Sauer R, Becker H, Hohenberger W, Rödel C, Wittekind C, Fietkau R, Martus P, Tschmelitsch J, Hager E, Hess CF, et al: Preoperative versus postoperative chemoradiotherapy for rectal cancer. N Engl J Med 351: 1731-1740, 2004.

3. Rödel C, Hofheinz R and Fokas E: Rectal cancer: Neoadjuvant chemoradiotherapy. Best Pract Res Clin Gastroenterol 30: 629-639, 2016.

4. Rödel C, Graeven U, Fietkau R, Hohenberger W, Hothorn T, Arnold D, Hofheinz RD, Ghadimi M, Wolff HA, Lang-Welzenbach M, et al: Oxaliplatin added to fluorouracil-based preoperative chemoradiotherapy and postoperative chemotherapy of locally advanced rectal cancer (the German CAO/ARO/AIO-04 study): Final results of the multicentre, open-label, randomised, phase 3 trial. Lancet Oncol 16: 979-989, 2015.

5. Aschele C, Cionini L, Lonardi S, Pinto C, Cordio S, Rosati G, Artale S, Tagliagambe A, Ambrosini G, Rosetti P, et al: Primary tumor response to preoperative chemoradiation with or without oxaliplatin in locally advanced rectal cancer: Pathologic results of the STAR-01 randomized phase III trial. J Clin Oncol 29: 2773-2780, 2011.

6. Gérard JP, Azria D, Gourgou-Bourgade S, Martel-Lafay I, Hennequin C, Etienne PL, Vendrely V, François E, de La Roche G, Bouché O, et al: Clinical outcome of the ACCORD 12/0405 PRODIGE 2 randomized trial in rectal cancer. J Clin Oncol 30: 4558-4565, 2012.

7. O'Connell MJ, Colangelo LH, Beart RW, Petrelli NJ, Allegra CJ, Sharif S, Pitot HC, Shields AF, Landry JC, Ryan DP, et al: Capecitabine and oxaliplatin in the preoperative multimodality treatment of rectal cancer: Surgical end points from National surgical adjuvant breast and bowel project trial R-04. J Clin Oncol 32: 1927-1934, 2014.

8. Iles S, Gollins S, Susnerwala S, Haylock B, Myint S, Biswas A, Swindell $\mathrm{R}$ and Levine E: Irinotecan+5-fluorouracil with concomitant pre-operative radiotherapy in locally advanced non-resectable rectal cancer: A phase I/II study. Br J Cancer 98: 1210-1216, 2008.

9. Mehta VK, Cho C, Ford JM, Jambalos C, Poen J, Koong A, Lin A, Bastidas JA, Young H, Dunphy EP and Fisher G: Phase II trial of preoperative 3D conformal radiotherapy, protracted venous infusion 5-fluorouracil, and weekly CPT-11, followed by surgery for ultrasound-staged T3 rectal cancer. Int J Radiat Oncol Biol Phys 55: 132-137, 2003.

10. Mohiuddin M, Winter K, Mitchell E, Hanna N, Yuen A, Nichols C, Shane R, Hayostek C and Willett C; Radiation Therapy Oncology Group Trial 0012: Randomized phase II study of neoadjuvant combined-modality chemoradiation for distal rectal cancer: Radiation therapy oncology group trial 0012. J Clin Oncol 24: 650-655, 2006.

11. Navarro M,Dotor E,RiveraF,Sánchez-RoviraP, Vega-Villegas ME, Cervantes A, García JL, Gallén M and Aranda E: A Phase II study of preoperative radiotherapy and concomitant weekly irinotecan in combination with protracted venous infusion 5-fluorouracil, for resectable locally advanced rectal cancer. Int J Radiat Oncol Biol Phys 66: 201-205, 2006.

12. Willeke F, Horisberger K, Kraus-Tiefenbacher U, Wenz F, Leitner A, Hochhaus A, Grobholz R, Willer A, Kähler G, Post S and Hofheinz RD: A phase II study of capecitabine and irinotecan in combination with concurrent pelvic radiotherapy (CapIri-RT) as neoadjuvant treatment of locally advanced rectal cancer. Br J Cancer 96: 912-917, 2007. 
13. Gollins S, Sun Myint A, Haylock B, Wise M, Saunders M, Neupane R, Essapen S, Samuel L, Dougal M, Lloyd A, et al: Preoperative chemoradiotherapy using concurrent capecitabine and irinotecan in magnetic resonance imaging-defined locally advanced rectal cancer: Impact on long-term clinical outcomes J Clin Oncol 29: 1042-1049, 2011.

14. Shirasaka T, Shimamato Y, Ohshimo H, Yamaguchi M, Kato T, Yonekura K and Fukushima M: Development of a novel form of an oral 5-fluorouracil derivative (S-1) directed to the potentiation of the tumor selective cytotoxicity of 5-fluorouracil by two biochemical modulators. Anticancer Drugs 7: 548-557, 1996.

15. Malet-Martino M and Martino R: Clinical studies of three oral prodrugs of 5-fluorouracil (capecitabine, UFT, S-1): A review. Oncologist 7: 288-323, 2002.

16. Shirao K, Ohtsu A, Takada H, Mitachi Y, Hirakawa K, Horikoshi N, Okamura T, Hirata K, Saitoh S, Isomoto H and Satoh A: Phase II study of oral S-1 for treatment of metastatic colorectal carcinoma. Cancer 100: 2355-2361, 2004.

17. Sadahiro S, Suzuki T, Tanaka A, Okada K, Kamijo A, Murayama C, Akiba T and Nakayama Y: Phase I/II study of preoperative concurrent chemoradiotherapy with S-1 for locally advanced, resectable rectal adenocarcinoma. Oncology 81: 306-311, 2011.

18. Sato T, Ozawa H, Hatate K, Onosato W, Naito M, Nakamura T, Ihara A, Koizumi W, Hayakawa K, Okayasu I, et al: A Phase II trial of neoadjuvant preoperative chemoradiotherapy with S-1 plus irinotecan and radiation in patients with locally advanced rectal cancer: Clinical feasibility and response rate. Int J Radiat Oncol Biol Phys 79: 677-683, 2011.

19. Matsusaka S, Ishihara S, Kondo K, Horie H, Uehara K, Oguchi M, Murofushi K, Ueno M, Mizunuma N, Shimbo T, et al: A multicenter phase II study of preoperative chemoradiotherapy with S-1 plus oxaliplatin for locally advanced rectal cancer (SHOGUN trial). Radiother Oncol 116: 209-213, 2015.

20. Marques RP, Duarte GS, Sterrantino C, Pais HL, Quintela A, Martins AP and Costa J: Triplet (FOLFOXIRI) versus doublet (FOLFOX or FOLFIRI) backbone chemotherapy as first-line treatment of metastatic colorectal cancer: A systematic review and meta-analysis. Crit Rev Oncol Hematol 118: 54-62, 2017.

21. Hardiman KM, Ulintz PJ, Kuick RD, Hovelson DH, Gates CM, Bhasi A, Rodrigues Grant A, Liu J, Cani AK, Greenson JK, et al: Intra-tumor genetic heterogeneity in rectal cancer. Lab Invest 96: 4-15, 2016.
22. Panczyk M: Pharmacogenetics research on chemotherapy resistance in colorectal cancer over the last 20 years. World J Gastroenterol 20: 9775-9827, 2014.

23. Greenhalgh TA, Dearman C and Sharma RA: Combination of novel agents with radiotherapy to treat rectal cancer. Clin Oncol (R Coll Radiol) 28: 116-139, 2016.

24. Lawrence TS, Blackstock AW and McGinn C: The mechanism of action of radiosensitization of conventional chemotherapeutic agents. Semin Radiat Oncol 13: 13-21, 2003.

25. Hill EJ, Nicolay NH, Middleton MR and Sharma RA: Oxaliplatin as a radiosensitiser for upper and lower gastrointestinal tract malignancies: What have we learned from a decade of translational research? Crit Rev Oncol Hematol 83: 353-387, 2012.

26. Zhu AX and Willett CG: Chemotherapeutic and biologic agents as radiosensitizers in rectal cancer. Semin Radiat Oncol 13: 454-468, 2003.

27. Leal F, Ferreira FP and Sasse AD: FOLFOXIRI regimen for metastatic colorectal cancer: A systematic review and meta-analysis. Clin Colorectal Cancer 16: 405-409.e2, 2017.

28. Jung M, Shin SJ, Koom WS, Jung I, Keum KC, Hur H, Min BS, Baik SH, Kim NK, Kim H, et al: A randomized phase 2 study of neoadjuvant chemoradiaton therapy with 5-fluorouracil/leucovorin or irinotecan/S-1 in patients with locally advanced rectal cancer. Int J Radiat Oncol Biol Phys 93: 1015-1022, 2015.

29. Hong YS, Kim DY, Lim SB, Choi HS, Jeong SY, Jeong JY, Sohn DK, Kim DH, Chang HJ, Park JG and Jung KH: Preoperative chemoradiation with irinotecan and capecitabine in patients with locally advanced resectable rectal cancer: Long-term results of a Phase II study. Int J Radiat Oncol Biol Phys 79: 1171-1178, 2011.

30. Sato T, Hayakawa K, Tomita N, Noda M, Kamikonya N, Watanabe T, Kato D, Sakai Y, Hiraoka M, Shimada M, et al: A multicenter phase I study of preoperative chemoradiotherapy with S-1 and irinotecan for locally advanced lower rectal cancer (SAMRAI-1). Radiother Oncol 120: 222-227, 2016.

31. Ishihara S, Matsusaka S, Kondo K, Horie H, Uehara K, Oguchi M, Murofushi K, Ueno M, Mizunuma N, Shinbo T, et al: A phase I dose escalation study of oxaliplatin plus oral S-1 and pelvic radiation in patients with locally advanced rectal cancer (SHOGUN trial). Radiat Oncol 10: 24, 2015. 